\title{
Yang-Mills ghost propagator in linear covariant gauges
}

\author{
Fabio Siringo \\ Dipartimento di Fisica e Astronomia dell'Università di Catania, INFN Sezione di Catania, \\ Via S.Sofia 64, I-95123 Catania, Italy
}

(Received 21 February 2019; published 17 May 2019)

\begin{abstract}
From first principles, using a screened expansion, a simple one-loop analytical expression is provided for the ghost propagator of pure SU(3) Yang-Mills theory in a generic linear covariant gauge. At variance with the Landau gauge, the ghost dressing function is suppressed in the infrared and vanishes at $p=0$, as predicted by other approaches in the continuum. However, in the very limited range where lattice data are available, no detectable deviation is found from the Landau gauge, thus reconciling some recent lattice data and previous continuum predictions.
\end{abstract}

DOI: 10.1103/PhysRevD.99.094024

Usually, in the IR, the gauge-fixed correlation functions of QCD and Yang-Mills theory are studied in the Landau gauge. The gauge-fixed Faddeev-Popov Lagrangian, which is the most direct way to quantize the theory in perturbation theory, is not easily implemented in lattice calculations except for the Landau gauge where the gauge parameter $\xi=0$. Even in the continuum, because of the breakdown of the standard perturbation theory, most of the available predictions rely on numerical calculations at $\xi=0$.

While a qualitative [1-4] and even quantitative [5,6] agreement has been reached for the gluon propagator at $\xi>0$, the behavior of the ghost propagator $G(p)$ has not been clearly established yet. There are strong analytical arguments for a suppression of the ghost dressing function $p^{2} G(p)$ in the IR [2,3,7], but there is no quantitative information on the entity of the suppression and on the scale at which it should be observed. On the other hand, recent lattice calculations [8] indicate that the ghost propagator is basically gauge parameter independent in the IR, at least at the energies and $\xi$ values explored so far. However, while that lattice approach to the Faddeev-Popov matrix seems to be very promising, the explored range might be too limited for any final conclusion to be drawn [8].

In this paper, it is shown that the recent lattice data of Ref. [8] are perfectly consistent with the outcome of the screened expansion [9-13], which yet predicts the vanishing of the ghost dressing function at $p=0$, as found in Ref. [2]. From first principles, at one loop, an analytical

Published by the American Physical Society under the terms of the Creative Commons Attribution 4.0 International license. Further distribution of this work must maintain attribution to the author(s) and the published article's title, journal citation, and DOI. Funded by SCOAP ${ }^{3}$. expression for the ghost dressing function of pure YangMills theory is obtained by the screened expansion in a generic linear covariant gauge, showing an evident suppression of the dressing function for small momenta $p<$ $0.5 \mathrm{GeV}$ and large values of the gauge parameter $\xi>0.1$, in qualitative agreement with previous calculations in the continuum [2,3]. Thus, the apparently contrasting predictions of lattice calculations and continuum approaches are reconciled by the present study, since the suppression of the ghost dressing function is only observed very deep in the IR, while no detectable deviation from the Landau gauge is found in the very limited range where the lattice data are available.

The screened expansion has been proposed in the last few years $[9,10]$ and shown to be a powerful tool for describing the analytic properties of the correlators of QCD in the IR [6,11]. The expansion arises from a change of the expansion point of ordinary perturbation theory, with the massless free gluon propagator, which is replaced by a screened massive propagator without changing the total action, providing explicit one-loop analytical expressions which are in excellent agreement with the lattice data $[6,12,13]$.

The screened expansion is very predictive since the mass emerges from a new expansion point of the exact FaddeevPopov Lagrangian, at variance with other massive one-loop calculations, e.g., Refs. $[14,15]$, where the mass parameter was added by hand. Having not modified the total action, the method does not contain any phenomenological parameter and is based on first principles [13]. Moreover, its extension to finite temperature $[16,17]$ and to a generic covariant gauge [6] has allowed the study of several features, like the gauge invariance and temperature dependence of the gluon dispersion relations, which cannot be observed by lattice calculations. In fact, like for ordinary perturbation theory, the extension to a generic linear 
covariant gauge is straightforward, and if the expansion is optimized by the constraints of the exact Becchi-RouetStora-Tyutin symmetry of the action, the gluon propagator can be evaluated for any value of the gauge parameter $\xi$, yielding reliable quantitative predictions even in the Feynman gauge, which has not been explored much yet [18-20]. For the gluon propagator, the agreement with the lattice is very good in the limited range $\xi<0.5$ where the data are available [5]. Thus, it would be now interesting to compare the recent data of Ref. [8] with the outcome of the screened expansion for the ghost propagator.

The ghost sector and the running coupling have been already studied by the screened expansion in a very recent work [13] where the general renormalization of the expansion is discussed in the Landau gauge and some general stationary conditions are derived for the optimization of the finite parts of the renormalization constants, from first principles. The analytical predictions of the expansion are found in excellent agreement with the lattice data of Ref. [21] for the correlators and for the strong coupling.

While that work was in the Landau gauge, the extension to a generic covariant gauge is trivial for the ghost propagator at one loop, since the gauge parameter enters through the longitudinal part of the gluon propagator of a unique one-loop graph [6]. The longitudinal part of the gluon propagator is known exactly and is equal to the free longitudinal part of ordinary perturbation theory. Thus, for a generic $\xi \neq 0$, the new self-energy contribution is just the same as that of perturbation theory. By dimensional regularization, for $d=4-\epsilon$, it reads

$$
\Sigma_{\xi}(p)=\xi \frac{N}{4}\left(\frac{\alpha_{s}}{4 \pi}\right) p^{2}\left(\frac{2}{\epsilon}+\ln \frac{\mu^{2}}{p^{2}}+C\right),
$$

where $\alpha_{s}=g^{2} /(4 \pi)$ is the strong coupling, $\mu$ is an arbitrary energy scale, $C$ is an arbitrary constant, and $p^{2}$ is the momentum in the Euclidean formalism. The diverging part gets canceled by the usual counterterm arising from wave function renormalization, so that the finite part of the total ghost self-energy can be written as

$$
\Sigma(p)=3 N\left(\frac{\alpha_{s}}{4 \pi}\right) p^{2}\left[\mathcal{G}(s)-\frac{\xi}{12} \ln s\right],
$$

where $s=p^{2} / m^{2}(\xi)$ and $m(\xi)$ is a gauge-dependent mass parameter which was determined in Ref. [6] by requiring that the poles of the gluon propagator are gauge parameter independent, as imposed by Nielsen identities. A very accurate interpolation of the curve $m(\xi)$ in the range $0<\xi<1$ was determined in [6]

$$
m^{2}(\xi) / m_{0}^{2} \approx 1-0.39997 \xi+0.064141 \xi^{2},
$$

where the mass parameter $m_{0}$ must be regarded as a phenomenological energy scale which fixes the units.
By a comparison with the lattice units of Ref. [21], the value $m_{0}=0.656 \mathrm{GeV}$ was extracted [6]. The adimensional function $\mathcal{G}(s)$ gives the finite part of the self-energy in the Landau gauge, and its explicit analytical expression has been reported in several papers [9-11,13],

$\mathcal{G}(s)=\frac{(1+s)^{2}(2 s-1)}{12 s^{2}} \ln (1+s)-\frac{s}{6} \ln (s)+\frac{1+2 s}{12 s}$.

Following the scheme of Ref. [13], up to a finite renormalization factor, the ghost dressing function reads

$$
p^{2} G(p)=\left[\mathcal{G}(s)-\frac{\xi}{12} \ln s+\mathcal{G}_{0}\right]^{-1},
$$

where the constant $\mathcal{G}_{0}$ depends on the renormalization scheme and can be optimized by the same stationary conditions of Ref. [13], yielding $\mathcal{G}_{0}=0.14524,0.1445$, $0.1392,0.1248$ at $\xi=0,0.1,0.5,1.0$, respectively, for $\mathrm{SU}(3)$. Thus, for a generic gauge, the variation of the optimal constant $\mathcal{G}_{0}$ is small and of order $\delta \mathcal{G}_{0} / \mathcal{G}_{0} \approx 0.1 \xi$. Moreover, by its definition, the sensitivity to any change is vanishing at the stationary point, so that the dependence of $\mathcal{G}_{0}$ on $\xi$ can be safely neglected at the scale of the figures shown below, where the Landau gauge value is used, $\mathcal{G}_{0}=$ 0.14524 [13].

By inspection of Eq. (5), we observe that the dressing function is logarithmically suppressed in the IR for any $\xi \neq 0$. Moreover, since $\mathcal{G}(s)$ is finite in the limit $s \rightarrow 0$ [13], the dressing function is zero at $p=0$, as predicted in Ref. [2]. In that work, because of the self-consistency of the equations, the leading behavior was $(\xi \ln p)^{-1 / 2}$, while, here, the simple one-loop dressing function vanishes at the stronger rate $(\xi \ln p)^{-1}$. Thus, we expect that in Eq. (5) the effect might be overestimated deep in the IR.

The SU(3) dressing function is shown in Fig. 1 for several values of the gauge parameter $\xi$ ranging from the Landau to the Feynman gauge. In the figure, the curves have an arbitrary renormalization at the scale $\mu=4 \mathrm{GeV}$. In the same figure, some large-volume lattice data of Ref. [21] are shown for the Landau gauge. The data have been renormalized in order to match the corresponding curve for $p<2 \mathrm{GeV}$. As discussed in Ref. [13], no comparison can be made above that point because the fixedcoupling one-loop dressing function needs to be corrected by renormalization-group effects.

The dressing function has a maximum for any $\xi \neq 0$, but the location of the maximum is at the very small momentum $p=0.064 \mathrm{GeV}$ for $\xi=0.1$, which is the largest $\xi$ value reached in Ref. [8]. The maximum moves to $p=$ $0.18 \mathrm{GeV}$ and $p=0.28 \mathrm{GeV}$ for $\xi=0.5$ and $\xi=1$, respectively. Even in the Feynman gauge, the maximum is far below $p=0.5 \mathrm{GeV}$, which is the smaller momentum in Ref. [8]. 


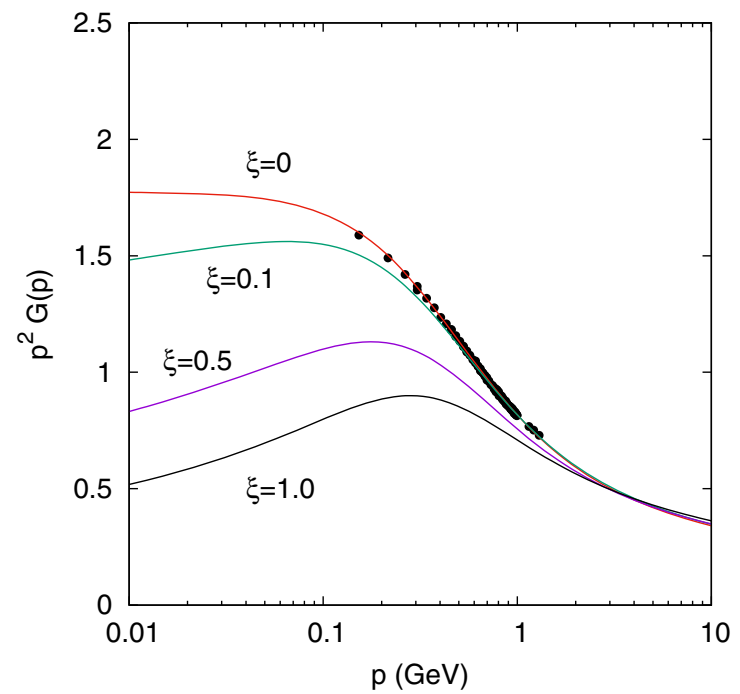

FIG. 1. The $\mathrm{SU}(3)$ ghost dressing function $p^{2} G(p)$ is evaluated by Eq. (5) for different values of $\xi$, ranging from the Landau $(\xi=0)$ to the Feynman gauge $(\xi=1)$, and plotted together with some lattice data of Ref. [21] in the Landau gauge $(\beta=6$, $L=80)$.

Above $p=0.5$, the curves are not distinguishable for $\xi \leq 0.1$, in perfect agreement with the preliminary results of Ref. [8] which show no detectable difference with the data in the Landau gauge. However, Fig. 1 shows that a difference should be observable in the lattice data if a smaller momentum $p \approx 0.2 \mathrm{GeV}$ is reached, or even at $p=$ $0.5 \mathrm{GeV}$ if a larger gauge parameter is taken (say, $\xi=0.5$ at least).

We must mention that the effect in Fig. 1 has been magnified by plotting the dressing function $p^{2} G(p)$ on a linear scale instead of the diverging propagator $G(p)$, which was shown on a log scale in Ref. [8]. Actually, as shown in Fig. 2, if the propagator is plotted on the same scale of Ref. [8], the differences are not detectable anymore, and even in the Feynman gauge, the curve follows the data of the Landau gauge. Thus, no conclusion can be drawn from the preliminary lattice data of Ref. [8] unless the authors can manage to show the dressing function, as in Fig. 1. It would be a very important test for the predictions of the screened expansion if still no difference could be observed in the data on that scale. Of course, if smaller energies or larger gauge parameters could be reached on the lattice by that new method, then a more complete test of the expansion would be available.

In summary, we have shown that the screened expansion provides a very simple one-loop expression for the ghost

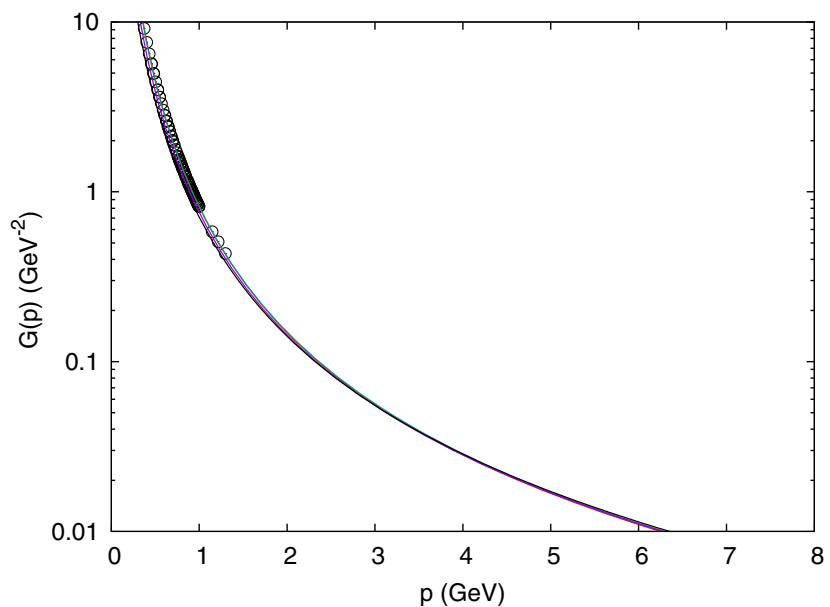

FIG. 2. The SU(3) ghost propagator $G(p)$ is evaluated by Eq. (5) and shown for the same values of the gauge parameter as in Fig. 1, together with the same lattice data in the Landau gauge, but on the log scale used in Ref. [8]. Observe the exchange of the $\log$ and linear scales, between the two axes, compared to Fig. 1.

propagator in a linear covariant gauge. The $\mathrm{SU}(3)$ dressing function and propagator have been shown for several values of the gauge parameter $\xi$, up to the Feynman gauge, and compared with the very recent lattice data of Ref. [8]. Even if the data seem to suggest that the ghost propagator is gauge parameter independent, we argue that no final conclusion can be drawn from the data because of the limited explored range of $\xi$ and $p$. In fact, while the analytical curves are in perfect agreement with the data in that range, they show an evident suppression of the ghost dressing function for smaller momenta $p<0.5 \mathrm{GeV}$ and larger values of the parameter $\xi>0.1$, in qualitative agreement with previous calculations in the continuum $[2,3]$. Moreover, we point out that in order to amplify the effect and make it observable the ghost dressing function should be plotted on a linear scale rather than the diverging propagator on a log scale. Otherwise, as shown in Fig. 2, no effect would be seen since the propagator still diverges even for $\xi \neq 0$, hiding the effect of the suppression in the IR. More extended data, by the method of Ref. [8], will be very welcome for providing a more stringent comparison with the present and previous analytical predictions.

\section{ACKNOWLEDGMENTS}

The author acknowledges a partial financial support from "Piano per la Ricerca di Ateneo 2017/2020-Linea di intervento 2" of the University of Catania. 
[1] M. A. L. Capri, A. D. Pereira, R. F. Sobreiro, and S. P. Sorella, Eur. Phys. J. C 75, 479 (2015).

[2] A. C. Aguilar, D. Binosi, and J. Papavassiliou, Phys. Rev. D 91, 085014 (2015).

[3] M. Q. Huber, Phys. Rev. D 91, 085018 (2015).

[4] A. Cucchieri, T. Mendes, and E. M. S. Santos, Phys. Rev. Lett. 103, 141602 (2009).

[5] P. Bicudo, D. Binosi, N. Cardoso, O. Oliveira, and P. J. Silva, Phys. Rev. D 92, 114514 (2015).

[6] F. Siringo and G. Comitini, Phys. Rev. D 98, 034023 (2018).

[7] M. A. L. Capri, D. Fiorentini, M. S. Guimaraes, B. W. Mintz, L. F. Palhares, S. P. Sorella, D. Dudal, I. F. Justo, A. D. Pereira, and R. F. Sobreiro, Phys. Rev. D 93, 065019 (2016).

[8] A. Cucchieri, D. Dudal, T. Mendes, O. Oliveira, M. Roelfs, and P. J. Silva, Phys. Rev. D 98, 091504(R) (2018).
[9] F. Siringo, arXiv:1509.05891.

[10] F. Siringo, Nucl. Phys. B907, 572 (2016).

[11] F. Siringo, Phys. Rev. D 94, 114036 (2016).

[12] F. Siringo, EPJ Web Conf. 137, 13016 (2017).

[13] F.Siringo, arXiv:1902.04110.

[14] M. Tissier and N. Wschebor, Phys. Rev. D 82, 101701(R) (2010).

[15] M. Tissier and N. Wschebor, Phys. Rev. D 84, 045018 (2011).

[16] F. Siringo, Phys. Rev. D 96, 114020 (2017).

[17] G. Comitini and F. Siringo, Phys. Rev. D 97, 056013 (2018).

[18] F. Siringo, Phys. Rev. D 90, 094021 (2014).

[19] F. Siringo, Phys. Rev. D 92, 074034 (2015).

[20] F. Siringo, Phys. Rev. D 88, 056020 (2013).

[21] A. G. Duarte, O. Oliveira, and P. J. Silva, Phys. Rev. D 94, 014502 (2016). 